\title{
La moralidad distribuida y la tecnología. Cómo las cosas nos hacen morales*
}

\author{
LORENZO MAGNANI \\ Departamento de Filosofía y Laboratorio de Filosofía de la Computación \\ Universidad de Pavía, Pavía, Italia; \\ Departamento de Filosofía, Universidad Sun Yat-sen \\ Guangzhou (Canton), P.R. China
}

\begin{abstract}
RESUMEN. En el presente artículo se sostiene que, a través de la tecnología, las personas podemos simplificar y resolver tareas morales incluso en presencia de información incompleta o de una capacidad insuficiente para la acción moral. Muchas cosas externas, normalmente concebidas como inertes desde un punto de vista moral, pueden considerarse lo que aquí se denominarán mediadores morales. Por lo tanto, no todas las herramientas morales están en el interior de nuestra cabeza, sino que muchas están distribuidas en objetos y estructuras externas que funcionan como dispositivos éticos. Con el objeto de investigar con detalle este conflicto ético entre los seres humanos y las cosas, consideraremos el papel que desempeñan los objetos, las estructuras y los artefactos tecnológicos mostrándolos como delegados y mediadores morales.
\end{abstract}

Palabras clave: ética y tecnología, moralidad distribuida, mediadores morales, mediadores epistémicos, imaginación moral, modelos externos artificiales.
ABSTRACT. In this paper, I contend that through technology people can simplify and solve moral tasks when they are in the presence of incomplete information and possess a diminished capacity to act morally. Many external things, usually inert from the moral point of view, can be transformed into what we will call moral mediators. Hence, not all of the moral tools are inside the head, many of them are shared and distributed in «external» objects and structures which function as ethical devices. Aiming at illustrating the intrigue of this ethical struggle between human beings and things, we will discuss the role of objects, structures, and technological artifacts by presenting them as moral carriers and mediators.

Key words: ethics and technology, distributed morality, moral mediators, epistemic mediators, moral imagination, artificial external models.

\footnotetext{
* Traducción de Paula Olmos (UNED) y Roberto Feltrero (IFS, CSIC)
} 


\section{La acción racional en el incierto mundo de los humanos}

Podríamos definir la moralidad, en último extremo, como «el empeño en guiar nuestra conducta por medio de la razón — es decir, en hacer aquello para cuya justificación hallamos mejores razones- teniendo en cuenta por igual los intereses de cada uno de los individuos que han de verse afectados por nuestra conducta: no debe haber privilegiados» (Rachels, 1999).

El razonamiento moral podría considerarse como una forma de anticipación de «mundos posibles», un modo de crear espacios de oportunidades para configurar el mundo de los humanos y actuar en él. Resulta útil para anticipar los riesgos, las posibilidades y las consecuencias de los actos humanos, y para adoptar o, en su caso, evitar toda una serie de pautas de conducta. Para ello, necesitamos contar con 1) principios/razones buenos y acertados que podamos aplicar a diversos problemas y que nos permitan elaborar argumentos para defender nuestra perspectiva moral frente a otros puntos de vista y 2) modos apropiados de razonamiento que nos posibiliten aplicar tales principios de la mejor forma posible. «Crear una ética» significa crear un mundo con instrucciones para enfrentarnos a distintos problemas y situaciones (bien sean reales o abstractos). Este proceso exige el concurso de cierta habilidad y creatividad para poder reaccionar ante casos previamente desconocidos o moralmente conflictivos. De este modo, los sucesos y situaciones pueden ser reinterpretados como oportunidades o, en su caso, situaciones de riesgo en las que aplicar nuevas instrucciones morales.

\section{Tratar a las personas como medios, respetar a las personas como a las cosas}

En los últimos tiempos, los seres no humanos, objetos y estructuras, como los artefactos tecnológicos y las máquinas han adquirido una nueva dimensión moral en virtud de sus valores intrínsecos. La tradición ética kantiana nos enseña que no debemos tratar a los seres humanos únicamente como «medios», o «cosas», es decir, de un modo meramente instrumental, sino que también debemos tratarlos como «fines». Aquí se defiende, sin embargo, que a los seres humanos se les puede tratar como «cosas», en el sentido de que deben ser «respetados» de igual manera que, a veces, respetamos a las cosas. Debemos reclamar para nosotros mismos los valores instrumentales y morales que ya asignamos a determinadas cosas y objetos externos.

Como es bien sabido, el imperativo categórico de Immanuel Kant prescribe «obra como si la máxima de tu acción pudiera convertirse por tu voluntad en una ley universal de la naturaleza». (Kant, 2002, p. 104). Al concretar «la fórmula del fin en sí mismo», Kant puntualiza en su Fundamentación para una metafísica de las costumbres que:

[...] el hombre, y en general todo ser racional, existe como fin en sí mismo, no simplemente como un medio para ser utilizado discrecionalmente por esta o aquella voluntad, sino que tan- 
to en las acciones orientadas hacia sí mismo como en las dirigidas hacia otros seres racionales el hombre ha de ser considerado siempre al mismo tiempo como un fin». (p. 114)

Tales consideraciones kantianas nos conducen al siguiente imperativo práctico: «obra de tal modo que uses a la humanidad, tanto en tu persona como en la de cualquier otro, siempre al mismo tiempo como un fin y nunca simplemente como un medio» (p. 116). «En el reino de los fines todo tiene o bien un precio o bien una dignidad. En lugar de lo que tiene un precio puede ser colocado algo equivalente; en cambio, lo que se halla por encima de todo precio y no se presta a equivalencia alguna, eso posee una dignidad» (p. 123-124). Las cosas que los seres humanos necesitan tienen un «precio comercial»; y aquellos artículos que responden más al deseo que a la necesidad tienen un «precio de afecto» (Affektionspreis). Pero «[...] aquello que constituye la condición para que algo sea fin en sí mismo, eso no tiene meramente valor relativo o precio sino un valor intrínseco, esto es, dignidad» (ibíd.)

Podemos invertir los términos de esta maravillosa lección kantiana: es posible otorgar a las cosas el trato o el respeto que normalmente reservamos a los seres humanos. Muchas cosas, o medios, que en principio carecen de valor, o que en principio se evalúan tan solo en términos de su precio de mercado o de su precio afectivo, pueden llegar a adquirir un estatuto moral o valor intrínseco. Y al contrario, así como a las cosas se les pueden asignar nuevos tipos de valor, también puede hacerse lo mismo con los seres humanos, ya que existen aspectos morales positivos en el tratamiento de las personas como cosas, tal como veremos a continuación ${ }^{1}$.

Ideas antropocéntricas como las que informan el imperativo kantiano, han hecho difícil el que las personas adquieran valores morales que normalmente se atribuyen a las cosas y también el que las cosas alcancen la valoración moral tradicionalmente reservada a las personas. Como ya hemos dicho, en términos kantianos, las personas no deben ser «tratadas como medios (y sólo como medios)». Aquí se propone la actualización de tal principio mediante uno nuevo: respetar a las personas como a cosas en sentido positivo. En este marco, se respeta a las personas como a «medios» de modo que se establece un círculo virtuoso en el que los aspectos morales positivos, presentes en las cosas, pueden invocarse al reconsiderar los dones morales que atribuimos a las personas.

Quizá una de las primeras «cosas» en obtener nuevos derechos morales dentro de la cultura occidental fueron las mujeres, una novedad que no gozó, ni mucho menos, en un principio, de aceptación universal. De hecho, las ideas que en este sentido proclamó Mary Wollstonecraft en su tratado de 1792 A Vindication of the Rights of Women se consideraron inicialmente absurdas (Singer, 1998).

${ }^{1}$ Para una exposición más clara de mi postura sobre las relaciones morales entre «personas» y «cosas» cf. (Magnani, cáp. 1, en prensa). 
Este tipo de conflicto ideológico se ha puesto de manifiesto una y otra vez en las últimas décadas cuando los defensores de los derechos de los animales y los teóricos de la ética medioambiental han tenido que desarrollar luchas similares a aquellas a las que las mujeres tuvieron que enfrentarse en el siglo XVIII: las que conlleva redefinir un medio como fin. Para alcanzar semejante objetivo, algunos intelectuales y activistas han intentado reformular el marco valorativo asociado, por ejemplo, a las plantas, animales y ecosistemas —incluso a la tierra - de modo que puedan ser considerados como «fines» y obtener los derechos y protecciones que se derivan de tal consideración. Tal como veremos en las siguientes secciones, los artefactos tecnológicos y las máquinas también se han redefinido como fines y han adquirido nuevas funciones morales.

Un ejemplo curioso de la significación de mi lema «respetar a las personas como a cosas» es el del caso notorio de lo que podríamos llamar el «yo quiero ser una especie peligro». Muchos se quejan de que las especies salvajes en peligro de desaparición reciben mayor protección legal y moral que las tradiciones culturales en similar proceso. La recientemente aprobada ley federal estadounidense de Derechos de los Artistas Gráficos de 1990 adopta, de hecho, el lenguaje del conservacionismo ecológico al conceder «derechos de atribución, integridad, y de prevención de la destrucción de objetos artísticos de reconocida valía para los creadores de obras de pintura, dibujo, grabado, escultura o fotografía» (Nagle, 1998). La importancia de esta analogía reside en el hecho de que algunas personas se consideran en peligro, precisamente porque no se sienten tratadas tan bien como algunas cosas (medios).

En lo que sigue, comentaremos algunas cuestiones éticas correspondientes a las relaciones entre «seres» $\mathrm{y}$ «cosas».

\section{Creando espacios para la ética}

Tanto los epistemólogos como los éticos enfatizan el papel de la imaginación ya sea en el razonamiento científico o en la deliberación y reflexión éticas. Incluso interpretando la «imaginación» como el simple proceso de recolectar y dar forma al conocimiento, se trataría de un proceso que da lugar a nuevas posibilidades cognitivas, ya que nos permiten ver las cosas como nunca antes las habíamos visto. Ver un «mundo moral» significa ver el mundo de un modo particular: la comprensión ética implica llegar a ver algunos aspectos de la realidad de un modo particular que influye en la acción humana dirigida a crear el futuro y a sobrevivir en él.

Johnson fija su atención en los procesos cognitivos que subyacen a la «imaginación moral». «Los principios morales desprovistos de imaginación moral se vuelven triviales, imposibles de aplicar e incluso suponen un obstáculo para la acción moralmente constructiva» (Johnson, 1956). Todo lo cual significa que el razonamiento analógico y el metafórico resultan ser muy importantes para la ética, 
por su capacidad para «reconceptualizar» las situaciones que se nos presentan. Por lo tanto, no debemos valorar negativamente el uso en las deliberaciones éticas de herramientas basadas en el uso de modelos ${ }^{2}$, considerando estos procedimientos como excesivamente subjetivos, flexibles y creativos que no se rigen por regla alguna ni aceptan la limitación de conceptos racionalmente definidos, lo que nos lleva a ver la imaginación como el enemigo de la moralidad. El papel de cierto tipo de actividad imaginativa basada en modelos es evidente, por ejemplo, en la Crítica de la razón pura en la que Kant aclara la importancia de los dispositivos mentales intermedios: los que capacitan a los seres humanos para relacionar los principios abstractos con el mundo real de la experiencia (cf. el caso del papel de la imaginación en la construcción geométrica). Al aplicar este discurso a las reglas morales, en su Crítica de la razón práctica Kant desarrolla la idea de que una regla moral pura (como máxima para la acción) se aplica a la experiencia concreta mediante una especie de «tipificación»: una especie de sustituto representativo (Kant, 2000). Esta tipificación puede interpretarse como una especie de visión figurativa de un mundo no existente, como medio para juzgar una situación moral dada. Kant niega que tal tipificación involucre a la imaginación, ya que mantiene que los juicios morales pertenecen a la razón pura práctica, pero según concluye Johnson «¿qué podría ser más imaginativo que esta forma de visión figurativa que se basa en una proyección metafórica?»(Johnson, 1993). Es esta clase de tipificación la que permite que la ética se vea beneficiada por la creación y utilización de este tipo de espacios de oportunidades. Pero ¿cómo se llega a esto?

Más allá de las reglas y de los principios, también los prototipos, los diagramas, los marcos conceptuales y las metáforas se convierten en vehículos del conocimiento moral basado en modelos que, en muchos casos, resultan verdaderamente eficientes al enfrentarse a problemas morales. Por ejemplo, la concepción gramatical de la moralidad representa un «prototipo» típicamente metafórico usado en ética; en ella, los principios gramaticales se entienden como análogos a los principios morales, tal y como se ejemplifica en el caso del paralelismo entre «hablar bien» y «actuar bien». Otro ejemplo sería la concepción metafórica de la acción como «movimiento» que nos lleva a la idea de que los principios morales serían reglas que nos marcan qué «caminos de acción» podemos tomar, cuáles tenemos necesariamente que tomar y cuáles debemos evitar (op. cit., p. 43). Al

2 En (Magnani, 2001) se introduce el concepto de razonamiento abductivo basado en modelos. El término «razonamiento basado en modelos» se utiliza para referirse a la construcción y manipulación de varios tipos de representaciones, no necesariamente proposicionales y/o formales, sino también mentales y/o correspondientes a mediadores externos. Podemos encontrar ejemplos de tales inferencias basadas en modelos en la construcción y manipulación de representaciones visuales, experimentos mentales y razonamientos analógicos. En este sentido, los sentimientos emocionales se pueden interpretar como un tipo de cognición basada en modelos. Y, por supuesto, el razonamiento abductivo — que es el razonamiento por hipótesis - puede llevarse a cabo por medio de modelos, de manera interna o con la ayuda de mediadores externos. Con ello me refiero a la actividad de producir hipótesis «morales» por medio de razonamientos abductivos basados en modelos. 
considerar las consecuencias de nuestras acciones y deliberaciones morales, esta visión de un mundo irreal que nos permite juzgar una acción propuesta puede llevarse a cabo por medio de un modelo.

En las siguientes secciones comentaremos cómo estas formas de comportamiento moral basadas en modelos se relacionan con lo que aquí se denominan «mediadores morales».

\section{Moralidad distribuida: delegar en ética, el papel de los mediadores morales}

En (Magnani 2001) se expone el razonamiento abductivo (razonamiento que obtiene hipótesis explicativas). En ese texto, se describe el papel —en la ciencia- de lo que denominamos «pensar haciendo», un concepto que sugiere que el razonamiento y los procesos inferenciales también poseen una interesante dimensión extrateórica. También las inferencias morales desempeñan un papel en la manipulación de determinados objetos externos y estructuras no humanas que actúan como elementos sustitutivos y complementarios del «sentir» y el «pensar» morales. En este caso, la delegación cognitiva en objetos externos, artefactos y máquinas es constitutivamente ética y puede relacionarse con la creación de lo que aquí se denominan mediadores morales.

La existencia de este modo de comportamiento cognitivo extra-teorético se nos revela en las múltiples situaciones cotidianas en que los humanos nos mostramos perfectamente capaces de ejecutar tareas muy eficaces (y habituales) sin poder, sin embargo, ofrecer, de manera inmediata, una explicación conceptual de nuestra acción. A veces, nos parece que la justificación conceptual para tales acciones estuvo en algún momento presente en nuestra memoria, aunque finalmente parece haberse disipado y es necesario reconstruirla; en otras ocasiones, tenemos que elaborar la justificación partiendo de cero, tal como sucede con el uso en ciencia de la manipulación abductiva en la creación de escenarios posibles.

Resulta difícil establecer un listado exhaustivo de invariantes del comportamiento que puedan considerarse resultado de este tipo de razonamiento ético manipulativo. La manipulación experta de objetos no humanos en contextos reales o artificiales exige que tanto nuevos como viejos patrones de comportamiento se ejecuten repetidamente de manera casi habitual. En lo que aquí respecta, nos referiremos tan solo excepcionalmente a acciones que siguen de manera simple y articulada un plan previamente establecido; lo que realmente nos interesa son las pautas de conducta implícitas e incorporadas a nuestro repertorio que aquí estamos denominando patrones tácitos. Esta clase de actividad moral «oculta» tiene un carácter tentativo: los patrones son hipótesis morales incorporadas que informan tanto nuestras conductas rutinarias como las nuevas y que, como tales, posibilitan cierto tipo de «hacer» moral. En algunas situaciones, los patrones de acción pueden seleccionarse entre aquellos que se encuentran almacenados en el sistema mente-cuerpo, como en caso de un niño 
que escucha a su hermanita pequeña llorando y que, sin pensarlo, automáticamente, trata de calmar al bebé acariciándole la cabeza o cantándole una nana, tal como tantas veces ha visto hacer a sus padres. En otros casos, es necesario crear patrones nuevos que nos permitan alcanzar determinados resultados morales.

Los patrones de comportamiento moral que enumeramos a continuación presentan algunos rasgos interesantes ${ }^{3}$ :

1. la sensibilidad ante aspectos curiosos o anómalos de una situación moral;

2. la sensibilidad a priori ante el carácter dinámico de la propia situación moral, y no sólo de las entidades involucradas o sus propiedades;

3. el recurso a todo tipo de manipulación que se beneficie de entornos creados artificialmente y de sentimientos inducidos desde el exterior para posibilitar la apertura a nuevas fuentes de información, de carácter estable y reproducible, relativas al conocimiento moral oculto y a las constricciones propias de cada situación. Este tipo de patrón se muestra claramente en, por ejemplo, las discusiones sobre el problema de la pena de muerte en las que utilizamos recursos como las estadísticas, la investigación científica, información que obtenemos de las entrevistas, etc., para poder contar con información real y no sesgada, como la que se refiere al alivio legítimo que sienten los familiares de una víctima de asesinato cuando el criminal es ejecutado. Sólo de este modo puede alcanzarse una reconfiguración del orden social de los grupos afectados 4 ;

4. ciertos ejemplos, ocasionales, de comportamiento moral espontáneo. Aquí se incluyen un grupo de patrones morales ciertamente comunes 5 ;

5. acciones morales espontáneas que se manifiestan en presencia de información incompleta o inconsistente o de una capacidad mermada para la actuación moral en el mundo. Este tipo de acciones tienen un alcance mayor que el correspondiente a un mero nivel «perceptual»;

6. la acción como control de los datos sensoriales, que nos muestra cómo podemos cambiar la posición del cuerpo (y/o de los objetos exteriores) para reconfigurar el orden social y las relaciones de la colectividad. También muestra cómo sacar partido de sucesos creados artificialmente para obtener determinados modos de estímulo. Este tipo de acciones nos proporcionan información táctil, visual, cinética, sentimental, emocional y corpórea que de otro modo no tendríamos disponible y que nos ayuda, por ejemplo, a cuidar a otras personas;

3 Tan solo se enumeran y se describen con algún detalle los patrones directamente relacionados con la construcción de mediadores morales. Para un tratamiento más amplio ver (Magnani, en prensa).

4 Sobre la reconfiguración del orden social tal y como se realiza en ciencia (en el laboratorio), cf. (Knorr-Cetina, 1999)

5 Podemos establecer una analogía entre estos patrones manipulativos y los utilizados en contextos epistémicos: cf. (Magnani 2001; Magnani 2002; Magnani y Dossena 2005). 
7. acciones que nos permiten construir nuevos modelos externos artificiales que encarnan mecanismos éticos y estructuras (como «instituciones», por ejemplo) y que sustituyen a sus correlatos «reales» y «naturales». (Téngase en cuenta, por supuesto, que tales estructuras «reales» y «naturales» también son artificiales: nuestro concepto cultural de «familia» no es en absoluto una institución natural). Por ejemplo, podemos reemplazar la estructura «natural» llamada «familia» por un entorno más adaptado a las necesidades del agente moral como sucede cuando, por ejemplo, sustraemos a un niño del cuidado de familiares maltratadores. En tales casos estamos recurriendo al uso de un «hogar» artificial para reconfigurar las relaciones. Un contexto diferente - un marco nuevo pero también artificial- posibilita la recuperación del niño y le permite reconstruir las percepciones morales dañadas por el maltrato. Obtenemos un efecto similar cuando trasladamos a personas con problemas de adicción a hogares colectivos en los que reciben tratamiento y apoyo. Podemos observar un ejemplo todavía más simple de ello en las estructuras externas que utilizamos cotidianamente para posibilitar los buenos modos y el comportamiento cortés: las vallas, los números que indican el turno en la panadería, los cordones que organizan las colas, etc.

Por supuesto, muchas de las acciones que llevamos a cabo para construir los modelos artificiales que hemos señalado no son tácitas sino explícitamente premeditadas y planificadas. Sin embargo, imaginemos a aquellos que crearon por primera vez estos artefactos (por ejemplo, a los fundadores de los hogares colectivos para personas con problemas de adicción). No es inverosímil pensar que tales artefactos se crearon principalmente «a través de la acción» (a través del diseño de nuevos patrones tácitos de acción moral) y no mediante la ejecución de proyectos predeterminados y bien contrastados. Muchas de las acciones que ejecutamos para construir artefactos tecnológicos y máquinas en las que pretendemos delegar nuestras decisiones morales (mediadores morales) pertenecen a esta categoría.

\subsection{Agentes y pacientes morales}

Los artefactos tecnológicos y las máquinas se diseñan, se producen, se distribuyen y se interpretan dentro del universo de los humanos; por ello, se encuentran específicamente interconectados con nuestras interacciones sociales: la tecnología afecta a aquello que las personas hacen y a cómo lo hacen. Por ejemplo, los ordenadores son agentes morales porque, 1) poseen cierto tipo de intencionalidad y 2) pueden provocar ciertos efectos en los llamados «pacientes morales», es decir, pueden perjudicar o beneficiar los intereses de los seres cuyos intereses son susceptibles de ser lesionados o favorecidos: «los artefactos son intencionales en la medida en que tienden a comportarse de un determinado modo cuando se le 
suministra un input de un cierto tipo. El diseñador de artefactos desempeña un papel complejo ya que si bien no puede negarse que las intenciones del diseñador están en sus artefactos, la funcionalidad de los mismos a menudo va más allá de lo que el diseñador haya podido prever o planificar. Tanto los inputs proporcionados por los usuarios como los outputs producidos por los artefactos pueden ser inesperados, imprevistos y hasta dañinos» (Johnson, 2004).

Algunos éticos sostienen que hay entidades que pueden interpretarse bien como pacientes morales, bien como agentes morales. No sólo los seres humanos, sino también las cosas, pueden considerarse pacientes morales (como entidades que pueden padecer tanto el bien como el mal) y también agentes morales (como entidades capaces de ejecutar acciones y que son fuente de acciones morales que, de nuevo, producen tanto el bien como el mal).

Habría, en principio, varios casos posibles:

1. que los dos conjuntos sean disjuntos (no habría entidades que fueran, al tiempo, agentes y pacientes, lo cual es muy poco plausible);

2. que el primer conjunto sea un subconjunto del segundo;

3. que los dos conjuntos posean una intersección; tanto el caso 2. como el 3. no resultan muy prometedores, ya que ambos prevén que exista al menos un agente moral que, en principio, no podría ser calificado como paciente moral (sólo un agente sobrenatural podría responder a tal definición, por ejemplo, un Dios que influya en el mundo sin verse afectado por él);

4. que toda entidad que cumpla los requisitos para ser agente, los cumpla también para ser paciente y viceversa (postura estándar), y, finalmente,

5. que todas las entidades que cumplan los requisitos para ser pacientes, los cumplan también para ser agentes ${ }^{6}$.

El hecho de que los animales parezcan cumplir las condiciones de los pacientes morales y que, sin embargo, no se consideren como agentes morales nos obliga a entender con matices la perspectiva 5. En resumen, ciertas «cosas» (entre ellas, las entidades artificiales ${ }^{7}$ ) amplían el conjunto de entidades que pueden verse involucradas en una situación moral, bien como agentes morales (por ejemplo, Internet) o bien como pacientes morales con valores intrínsecos (por ejemplo, una obra de arte). Por supuesto que la propiedad atribuida a las «cosas» y que les otorga el ser agentes o pacientes morales, no es exactamente la misma propiedad atribuida a los seres humanos.

${ }^{6}$ (Floridi y Sanders, 2003). Carstein-Stahl (2004) ha investigado recientemente si los ordenadores pueden considerarse agentes morales autónomos. Como los ordenadores no pueden comprender la información que almacenan y procesan, carecen de la capacidad básica para «reflexionar moralmente sobre algo». Discute este asunto presentando un curioso e interesante test llamado «el test moral de Turing».

${ }^{7}$ Sobre la extensión legal de la personalidad a los agentes artificiales (por ejemplo, los portales de venta a través de Internet) cf. las interesantes conclusiones del reciente (Chopra y White, 2003). Aquí se discuten también algunos temas de gran actualidad sobre los contratos suscritos por agentes artificiales y sobre sus consecuencias financieras y penales. 
Considero, sin embargo, que esta distinción entre pacientes y agentes morales, ciertamente correcta y útil, pasa por alto los aspectos dinámicos que quedan reflejados en la perspectiva, aquí defendida, basada los conceptos de delegación y externalización moral. La delegación moral en objetos externos y artefactos no tiene lugar por el hecho de que una determinada cosa posea, supuesta e intrínsecamente un grupo de propiedades que se consideran inherentes. Por ejemplo, la Gioconda no tiene libre albedrío ni intenciones propiamente dichas. Sin embargo, el modo en que interactúa dinámicamente con los seres humanos y el modo en que éstos le responden, es lo que le otorga valor. En este sentido, la concepción aquí defendida difiere de la que discrimina entre pacientes y agentes morales.

De acuerdo con dicha perspectiva, la Gioconda (o, para el caso, un sistema de venta a través de Internet) sería un paciente moral porque no posee todos los rasgos compartidos por (o que supuestamente comparten) los seres humanos (voluntad consciente, un verdadero libre albedrío, intenciones propiamente dichas, etc.). Sin embargo, esta explicación no da cuenta del proceso mediante el cual habitualmente delegamos en, y otorgamos valor (moral) a, las cosas que nos rodean. Por ejemplo, ¿cómo podría explicar la distinción agente-paciente la razón por la cual el primer regalo recibido de una novia puede llegar a adquirir un gran valor (intrínseco)? Puede que no sea más que una vieja y raída camiseta, pero, ciertamente, no importa.

Es más, existe aún otra razón para preferir la concepción basada en la delegación tal y como aquí se describe. La idea de que algunos artefactos y máquinas deberían ser respetados o deberían tener derechos propios se basa en la pretensión de que desempeñan importantes procesos cognitivos, a menudo dotados de gran valor instrumental y económico. Son pacientes morales y como tales pacientes deben ser respetados. De acuerdo con la perspectiva aquí defendida, ello es el resultado de una mediación moral. Cuando delegamos en las máquinas nuevas funcionalidades morales, podemos utilizarlas para describir nuevos rasgos morales de la cognición anteriormente imprevistos, lo que hace que, para los seres humanos, adquieran mayor valía y una funcionalidad más extensa. Algunas máquinas pueden desempeñar el papel de mediadores morales porque median en nuevos aspectos de la vida moral de los seres humanos ${ }^{8}$.

La distinción paciente-agente desvela ulteriores distinciones: resulta obvio que la agencia moral de los ordenadores no es la misma que la de los seres humanos $\mathrm{y}$, en este sentido, no difiere de la de otras tecnologías. Se ha argumentado que los ordenadores tienen cierto tipo de intencionalidad externa (como la que se expresa a través de realidades extra corpóreas, como actos del habla, frases escritas, mapas y otros artefactos diseñados por el hombre) pero que no pueden tener intencionalidad interna: su agencia puede compararse a la que, entre los seres humanos, se ejerce por subrogación, como la correspondiente a los inspectores

${ }^{8}$ Detallaremos este punto en la subsección «Mediadores Morales». 
fiscales o a los albaceas testamentarios (Powers, 2004). Todo ello muestra el carácter moral de los sistemas informáticos, ya que éstos presentan cierto tipo de intencionalidad y provocan efectos en pacientes morales, por lo que son objetos apropiados para la evaluación moral. En este caso, nos enfrentamos a lo que podríamos llamar una «moralidad sin mente» (Floridi y Sanders, 2003). El problema de la agencia moral de los artefactos también nos obliga a considerar políticas adecuadas mediante las cuales podríamos (y/o deberíamos) «castigarlos», es decir, censurarlos, modificarlos, rediseñarlos o eliminarlos.

Considero que este concepto extendido de «mediador moral» podría englobar y explicar de un modo más adecuado los temas planteados: los pacientes y agentes morales serían casos especiales de la categoría de mediadores morales.

\subsection{Mediadores morales}

Las consideraciones del punto anterior nos demuestran que una parte significativa de nuestras manipulaciones se emplea en construir una nueva fuente de información y conocimiento de gran alcance: los mediadores morales externos. Dicha expresión se construye sobre la de «mediadores epistémicos», un concepto que ya introduje en un texto anterior (Magnani, 2001, cap. 3) y que se refiere a las representaciones externas, objetos y artefactos que son relevantes para el descubrimiento científico y los procesos de razonamiento. Tal como se ha mencionado, los mediadores morales encarnan una especie de redistribución del esfuerzo moral a través de un procesamiento de objetos y de información que nos permite superar la insustancialidad y el carácter insatisfactorio de las acciones morales inmediatas o que somos capaces de hallar internamente (por ejemplo, principios, prototipos, etc.). Considero que el análisis de los mediadores morales nos puede ayudar a explicar los mecanismos de «el fenómeno macroscópico, creciente, por el cual las acciones morales globales y las responsabilidades colectivas emergen como resultado de la «mano invisible» de las interacciones sistémicas entre diversos agentes a nivel local» (Floridi y Sanders, 2003).

Además de ser el medio por el que modificamos el mundo para obtener objetivos deseables, cualquier acción tiene también una función moral: decimos que cuando las personas no tienen información adecuada o carecen de la capacidad para actuar moralmente en el mundo, pueden reestructurar su mundo con el objeto de simplificar y resolver las tareas morales. Los mediadores morales se usan también para evitar las constricciones latentes en el entorno humano. Las conexiones que somos capaces de descubrir nos proporcionan acceso a nueva y apreciable información ética. Por ejemplo, imaginemos una esposa cuyo trabajo le obliga a permanecer largas horas lejos de su marido, de modo que sus frecuentes ausencias resultan conflictivas para su relación. Espontáneamente, la mujer decide empezar a pasar más tiempo y de mayor calidad con su marido, en un intento de salvar su matrimonio. El efecto mediador de su acción espontánea puede dar lugar a que determinadas variables puedan verse afectadas por sucesos «imprevistos» y 
«positivos» en la relación y se modifiquen conjuntamente con otras variables informativas, sentimentales, sexuales, emocionales y, en términos generales, corpóreas.

Antes de que la pareja adoptase este nuevo y reconfigurado orden «social» - es decir, antes de que pasasen más tiempo juntos- no existía una conexión clara entre estas variables ocultas y las explícitas, y descubrir tales conexiones es algo que aporta una nueva e importante información: lo que en nuestro ejemplo podría ser un placer sexual inesperado y renovado, un acuerdo intelectual sorprendente o una inusitada coincidencia emocional en asuntos concretos.

Los fenómenos naturales también pueden servirnos de mediadores morales artificiales y externos: cuando, en los puntos anteriores, consideramos el principio de «respetar a las personas como a las cosas» nos estábamos refiriendo a la habilidad de los objetos externos «naturales» de establecer oportunidades para la adquisición de conocimiento ético, como sucede en el caso de las especies en peligro de extinción: aprendemos algo nuevo al ver cómo las personas tratan de redefinirse como «en peligro de extinción». Muchas cosas externas que tradicionalmente se han considerado moralmente inertes podrían verse transformadas en mediadores morales. Por ejemplo, podemos utilizar a los animales para identificar rasgos morales previamente desconocidos de los seres humanos en otras criaturas vivas, y lo mismo podríamos hacer con la tierra y con las entidades (no naturales) culturales. También podemos utilizar «herramientas externas» como la escritura, la narrativa, los ritos y toda clase de instituciones relevantes para reconfigurar los órdenes sociales insatisfactorios. Por lo tanto, no todas las herramientas morales están en el interior de nuestra cabeza; muchas están distribuidas en objetos y estructuras externas que funcionan como dispositivos éticos.

Los mediadores morales externos funcionan como los componentes de un sistema de memoria que atraviesa los límites entre las personas y su entorno. Por ejemplo, son capaces de transformar las tareas en manipulaciones simples que posibilitan nuevas inferencias morales en el nivel del razonamiento abductivo basado en modelos 9 . Cuando se traslada a un niño maltratado a un nuevo hogar para reconfigurar sus relaciones sociales, este nuevo mediador moral puede ayudarle a experimentar nuevas inferencias basadas en modelos - nuevas hipótesis cognitivas basadas en modelos- (por ejemplo, nuevas emociones respecto de los adultos y una nuevas visión respecto de su pasado maltrato).

Es más, podemos alterar nuestra experiencia corpórea del dolor a través de la acción, siguiendo la pauta del control de los datos sensoriales, tal como apuntamos anteriormente, es decir, modificando —inconscientemente- la posición del cuerpo y cambiando las relaciones con otros seres humanos y no

\footnotetext{
${ }^{9}$ Cf. nota 2 .
} 
humanos que experimentan la aflicción. El rico sentimiento moral y personal de la Madre Teresa y su consideración del dolor se configuran mediante la cercanía a las personas hambrientas y miserables, y a través de la manipulación de sus cuerpos. Para muchas personas, el entrenamiento moral se relaciona a menudo con este tipo de manipulación espontánea (y «afortunada») de sus propios cuerpos y datos sensoriales, de modo que construyen su moral de manera inmediata y no reflexiva, «haciendo».

Los artefactos, por supuesto, desempeñan el papel de mediadores morales de muy diversas maneras. Consideremos algunos de los efectos que tiene en la privacidad la mediación de ciertas máquinas. Más allá del apoyo de la escritura, las comunicaciones telefónicas y los medios de comunicación, gran parte de las interacciones humanas se hayan significativamente mediadas (y potencialmente registradas) a través de Internet. ¿Qué sucede entonces con el concepto de identidad tan relacionado con el concepto de libertad? A partir de ahora, la identidad debe interpretarse en un sentido más amplio: la cantidad de datos almacenados externamente, información, imágenes y textos relacionados con cada uno de nosotros como individuos es enorme. Este almacenaje de la información crea para cada persona una especie de «sombra de datos» externa que, junto con su cuerpo biológico, forma un «cyborg» de datos carnales y electrónicos que, real o al menos potencialmente, nos identifica. Considero que este nuevo y complejo «ser informacional» delimita una nueva ontología que, a su vez, trae consigo nuevos problemas morales. Ya no podemos aplicar las viejas reglas morales y los argumentos pasados de moda a seres que son, al mismo tiempo, biológicos (concretos) y virtuales, situados en un espacio local tridimensional $\mathrm{y}$, al tiempo, potencialmente «omnipresentes globalmente» como paquetes de información. Por ejemplo, ya no es fácil precisar dónde nos hallamos localizados desde el punto cibernético, y el incremento de las tecnologías de «telepresencia» lo dificulta aún más. Parece claro que los recursos externos no biológicos contribuyen a construir nuestra sensación variable de quién y qué somos, y de qué somos capaces de hacer. En (Magnani, en prensa) se presentan otros ejemplos relacionados con artefactos, computacionales y de otro tipo, como mediadores morales.

A lo largo de la historia, se ha considerado que las mujeres otorgan un mayor valor a las relaciones personales que los hombres, y por ello se las ha supuesto más capacitadas para actuar en situaciones relacionadas con la intimidad y el cuidado. Parecería que la orientación moral básica de las mujeres fomenta el cuidado tanto de personas, como de objetos externos a través de actos de carácter personal y particular, más que la relación con los demás mediada por una preocupación humanitaria general y abstracta. La ética del cuidado no considera esencial la «obligación» abstracta; es más, no requiere que apoyemos de manera imparcial los intereses de todos por igual. Más bien, se centra en relaciones a pequeña escala con personas y objetos externos de modo que, por ejemplo, no es tan importante «pensar» en ayudar a los niños 
desfavorecidos en todo el mundo (como tratan de hacer los hombres) como «hacerlo» cuando se nos requiere en cualquier lugar ${ }^{10}$.

Por lo tanto, «el cuidado» es un modo relevante de considerar a las personas y a las cosas y, como forma de moralidad llevada a cabo «haciendo», alcanza el nivel de un modo fundamental de inferencia y conocimiento morales. Respetar a las personas como a cosas es una extensión natural de la ética del cuidado. Una persona que trata a los objetos caseros «no humanos» con esmero, por ejemplo, es más probable que también trate a los seres humanos de un modo similar. Por consiguiente, incluso un humilde recipiente de cocina puede considerarse un mediador moral en el sentido que aquí se da a este concepto cognitivo.

Cuando limpio mi ordenador, me preocupo por él, por su valor económico y por su funcionalidad como herramienta para otros seres humanos. Cuando, por otro lado, utilizo mi ordenador como un mediador epistémico o cognitivo, para mi investigación o mis actividades didácticas, estoy teniendo en cuenta su valor intelectual como instrumento de apoyo. Para defender el lema de respetar a las personas como respetamos a los ordenadores, podemos recurrir a las características que los seres humanos compartimos con estas máquinas: 1) los seres humanos somos «herramientas» — biológicas - con valores económicos e instrumentales y como tales podemos ser «utilizados» para enseñar e informar a otros, de manera similar a como usamos el hardware y el software; por lo tanto, los seres humanos somos instrumentalmente valiosos para el resto de los seres humanos por compartir habilidades de distinto tipo; y 2) como los ordenadores, las personas somos habilidosos «solucionadores de problemas» dotados del valor intrínseco y moral de la cognición.

\section{Conclusiones}

La tesis principal defendida en este artículo es que, en los últimos tiempos, los seres no humanos, los objetos y las estructuras como los artefactos tecnológicos y las máquinas han llegado a adquirir una nueva valía moral y valor en sí mismos. La tradición kantiana en ética nos enseña que no debemos tratar a los seres únicamente como a «medios»o «cosas», es decir, de un modo meramente instrumental, sino que también debemos tratarlos como «fines». Considero, por el contrario, que los seres humanos pueden ser tratados como «cosas» en el sentido de que deben ser «respetados» tal como lo son a veces las cosas. Las personas deben reclamar los valores morales e instrumentales que ya se atribuyen a las cosas externas y a

10 Es más, tanto el escepticismo feminista en ética como el llamado «modelo expresivo-colaborativo» de moralidad contemplan la vida moral como «una negociación continua entre las personas, una práctica situada socialmente para el reparto mиtuo, la asunción o dejación de responsabilidades importantes y la comprensión de las implicaciones que éstas conllevan» (Urban-Walker, 1996, p. 276). Por supuesto, esta idea contrasta claramente con la llamada «concepción teórico-jurídica de la moralidad». 
los objetos. Ello resulta esencial para el objetivo de reconfigurar la dignidad humana en un mundo tecnológico como el nuestro. Con el objeto de investigar en detalle este conflicto ético entre los seres humanos y las cosas hemos considerado el papel que desempeñan los objetos, las estructuras y los artefactos tecnológicos mostrándolos como delegados y mediadores morales. Sostenemos que esta perspectiva puede resultar muy fructífera en el tratamiento de otros problemas similares sobre las relaciones entre las máquinas y la ética.

\section{BIBLIOGRAFÍA}

C.B. CARTESin-Stahl, «Information, ethics, and computers. The problem of autonomous moral agent», Minds and Machines, 14, pp. 67-83, 2004.

S. Chopra AND L. White, «Artificial agents. Personhood in law and philosophy». En R. López de Mántaras y L. Saitta, eds., Proceedings of the 16th European Conference on Artificial Intelligence, pp. 635-639, Amsterdam, 2003.

L. Floridi AND J.W. SANDERS, «The method of abstraction». En M. Negrotti, ed., Yearbook of the Artificial. Nature, Culture, and Technology. Models in Contemporary Sciences, Bern, 2003.

— «On the morality of artificial agents», Minds and Machines, 14, pp. 349-379, 2004.

M. Johnson, Moral Imagination. Implications of Cognitive Science for Ethics. The Chicago University Press, Chicago, IL, 1993.

D.G. Johnson, «Integrating ethics and technology». 2004. European Conference Computing and Philosophy, E-CAP2004, June 2-5, Pavia, Italy. Abstract.

I. Kant, Crítica de la razón práctica. Madrid, Alianza Editorial, 2000. Edición de Roberto R. Aramayo, (publicado originalmente en 1788).

- Fundamentación para una metafísica de las costumbres, Alianza Editorial, 2002. Edición de Roberto R. Aramayo (publicado originalmente en 1785).

K. Knorr-Cetina, Epistemic Cultures. How Sciences Make Knowledge. Harvard University Press, Cambridge, MA, 1999.

L. Magnani And R. Dossena, Perceiving the infinite and the infinitesimal world: unveiling and optical diagrams and the construction of mathematical concepts, 2005. En prensa en Foundations of Science.

L. Magnani, Abduction, Reason, and Science. Processes of Discovery and Explanation. Kluwer Academic/Plenum Publishers, New York, 2001.

— «Epistemic mediators and model-based discovery in science». En L. Magnani y N. J. Nersessian, eds., Model-Based Reasoning: Science, Technology, Values, pp. 305-329, New York, 2002. Kluwer Academic/Plenum Publishers.

- Knowledge as a Duty. Distributed Morality in a Technological World. en prensa.

J.C. NAGLE, «Endangered species wannabees», Seton Hall Law Review, 29:235-55, 1998.

T.M. POWERS, «Intentionality and moral agency in computers». 2004. European Conference Computing and Philosophy, E-CAP2004, June 2-5, Pavia, Italy. Resumen 
J. Rachels, The Elements of Moral Philosophy. McGraw Hill College, Boston Burr Ridge, IL, 1999.

P. SINGER, «All animals are equal». Philosophic Exchange, 1(5), pp. 243-57, 1998. También publicado en M.E. Zimmerman, J.B. Callicott, G. Sessions, K.J. Warren, y J. Clark, eds, Environmental Philosophy. From Animal Rights to Radical Ecology, pp. 26-80, Upper Saddle River, NJ. Prentice-Hall.

M. URBAN-WALKER, «Feminist skepticism, authority and transparency». En W. Sinnott-Armstrong y M. Timmons, eds, Moral Knowledge? New Readings in Moral Epistemology, pp. 167-292, Oxford, 1996. 